\title{
A Maximum Power Point Tracking Control for Small-Scale Wind Generator Based on Fuzzy Control
}

\author{
Yujie Ren \\ Department of Automation, North China Electric Power University, Baoding 071000, China.
}

Keywords: Small-Scale wind power generation; MPPT in variable step; fuzzy control.

\begin{abstract}
In order to improve the maximum power tracking control of a small permanent magnet wind generator, an improved scheme based on fuzzy control is proposed in this paper after analyzing the composition and tracking principle of the actual small-scale generator. The experiment shows that the variable step length control can be used to solve the uncertainty and randomness of wind speed in the generator set. And the scheme achieves a higher wind power system to improve the efficiency of energy utilization, also has good practical significance.
\end{abstract}

\section{Introduction}

Nowadays, the development speed of wind power industry is accelerating. The development of industry related technology is a difficult problem we need now. Small wind turbines are mainly used in areas without power grid popularization and the area is rich in wind resources. However, due to the strong randomness and instability of wind energy, the energy conversion efficiency of wind turbines produced by the existing technology level is too low. Improving the wind energy utilization rate of wind power generation under the existing technology has become a hot issue in China's wind power generation. There is a contradiction between tracking efficiency and power stability in traditional step parameter-perturbation observation control. The key to this problem is the selection of disturbance step size. In the article, the author analyzes the advantages and disadvantages of the traditional fixed step control scheme and proposes a new variable step size control scheme based on fuzzy control to improve the traditional scheme. This scheme can improve the rapidity and stability of the tracking scheme. It has better practical guiding significance to improve the actual utilization rate of wind energy.

\section{System Modeling}

Modeling of dynamic characteristics of wind generator:

$$
P m=\frac{1}{2} \rho \pi R^{2} C p(\beta, \gamma) V^{3}
$$

Interpretation: The density of the air around the wind turbine is $\rho$, its unit is $\mathrm{kg} / \mathrm{m}$. The speed of the external natural wind is $\mathrm{V}$, its unit is $\mathrm{m} / \mathrm{s}$. The radius of the impeller of a wind turbine is $\mathrm{R}$, its unit is $\mathrm{m}$. The mechanical power of wind turbines after absorbing wind energy is Pm, its unit is W. $\beta$ is the pitch angle of the blade; $r$ is the tip speed ratio.

Ordinary differential equations of permanent magnet generator in $\mathrm{d}-\mathrm{q}$ rotating coordinate system:

Under the condition of surface permanent magnet for wind power generator,

$$
\mathrm{T}_{e}=1.5 P \psi_{f} i_{q}
$$

Interpretation: shaft current of $\mathrm{Q}$ is iq. The magnetic chain of the rotor permanent magnet is $\Psi f . \mathrm{P}$ is the polar logarithm of the motor. According to Bates's theory, the maximum utilization coefficient of wind energy is about 0.59 under ideal circumstances. [2]

\subsection{Maximum Power Point Tracking Control}

The author mainly studies the method of maximum power point tracking for fixed pitch wind turbines. As the efficiency of wind energy conversion is the best only when the blade tip speed ratio is at the best, the energy conversion efficiency of the system can be improved by ensuring that the fans 
work at the best optimum condition.Fig. 1 is the characteristic curve of the output power and speed of the wind generator.

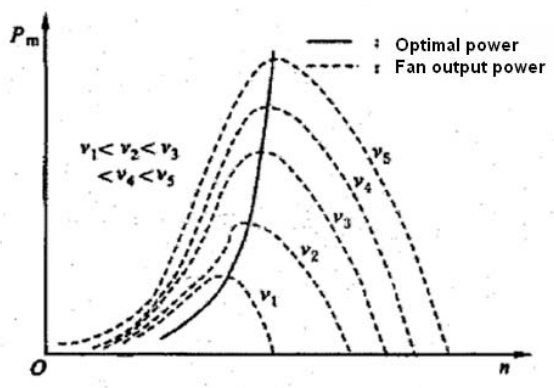

Fig. 1. Power performance curve of generator

Adjusting the duty ratio of the Boost converter is equivalent to changing the load characteristics of the generator. The change of the duty cycle also changes the speed of the generator. Tip speed ratio varies with generator speed. Duty cycle affects the wind energy utilization coefficient of the system until the end. [3] If we can always track the maximum power of the current time, we can greatly improve the efficiency of energy conversion. Wind turbines can obtain maximum wind energy utilization coefficient only when operating at the optimum tip speed ratio, and the output power Pm is also the largest. [4]

\subsection{Traditional Step Parameter-Perturbation Observation Control}

By applying the speed disturbance and comparing the measured power signals, the author can find the maximum power point of the wind turbine by changing the direction of the speed disturbance. And the disturbance step is constant in this process. There are two main problems in the fixed step MPPT control:

the fixed step will lengthen the search time of the generator with large inertia characteristics.

The constant step will produce the change instruction of the generator speed change, which makes the speed of the small wind generator change suddenly. It is not conducive to the stability of the system and the lifespan of the fan.

\subsection{Variable Step Disturbance Observation Based on Fuzzy Control}

\subsubsection{The Fuzzification of Input and Output}

In this paper, the change rate of power and duty ratio are chosen as input of fuzzy controller. The output is the disturbance step of the duty ratio. The quantification of input and output is quantified and reflected in the fields of fuzzy control, which are Ek, Edk, Eu. They all have 7 fuzzy sets: \{NB, NM, NS, ZO, PS, PM, PB\}.

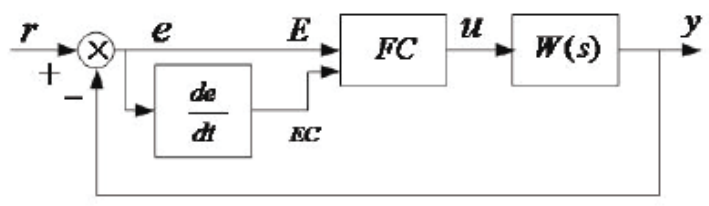

Fig. 2. The Principle of Fuzzy Control

Taking into account the actual characteristics of the small-scale wind generator system, its fuzzy decision rules are formulated as follows:

if the power continues to increase, the direction of the step length will be maintained, and the direction of the step length will be changed if the power decreases.

When the maximum power point is far away, the step length is increased; the step length is reduced when the maximum power point is closer.

This rule can be expressed in formula (3):

$$
\bar{u}=-\frac{\bar{e}+\overline{e c}}{2}
$$


That is, the output of the fuzzy controller is equal to the average of error and error rate.In order to adapt to different control model, the equilibrium factor $\alpha$ is introduced into the above formula, and an improved fuzzy rule is obtained.

$$
\overline{\mathrm{u}}=-[\alpha \bar{e}+(1-\alpha) \overline{e c}] \quad \alpha \in(0,1)
$$

The optimal balance factor can be obtained by using the optimization principle, and the optimal control scheme can be obtained. The center of gravity method is chosen by non-fuzzy processing to solve the fuzzy.

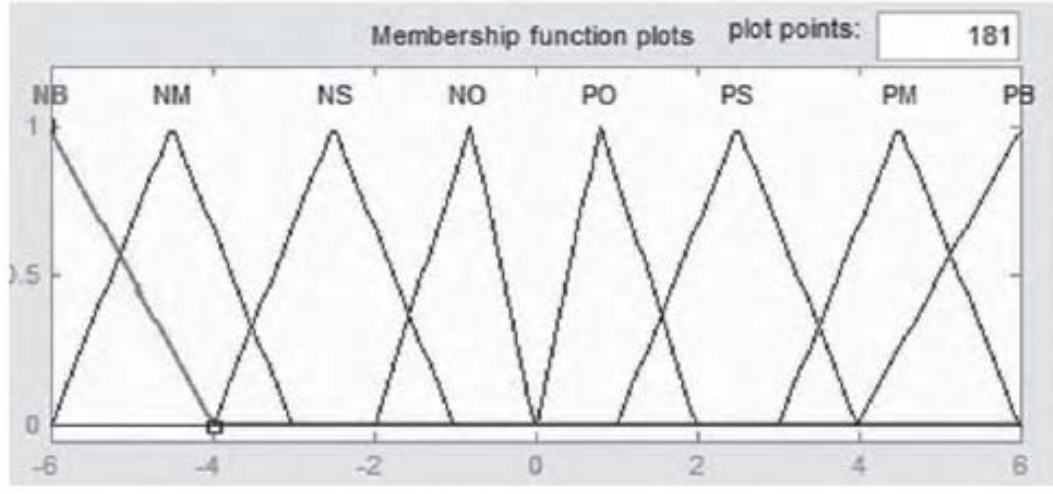

Fig. 3. Power Error Membership Function

Explanation: the closer the curve to the origin, the slower the farther away from the origin.

\subsubsection{Parameter Setting and Experiment}

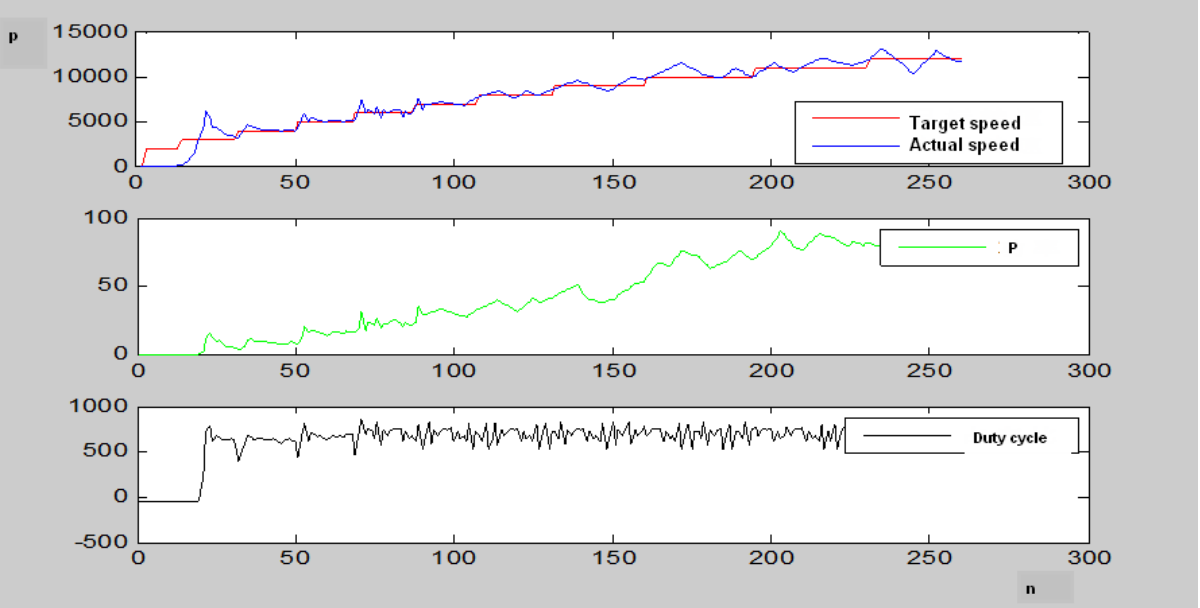

Fig.4. Maximum Power Point Tracking Curve with disturbance step size of 1000 and $\alpha=0.5$

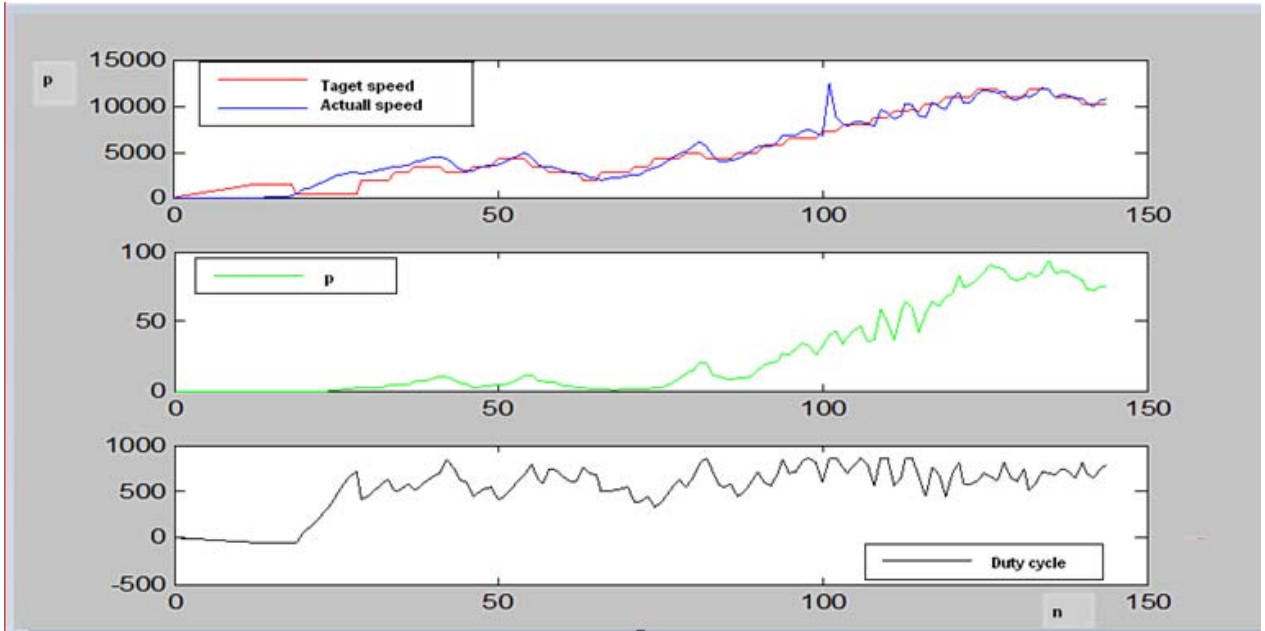

Fig.5. Maximum Power Point Tracking Curve with disturbance step size of 750 and $\alpha=0.3$ 


\section{Conclusion}

The variable step MPPT scheme under fuzzy control solves the disadvantage of fixed search step and makes the speed command change very quickly and achieves maximum power point tracking. Reducing the search step can improve the power oscillation phenomenon of the generator near the maximum power point, thereby reducing unnecessary energy consumption. When the model is far away from the maximum power point, it should improve the step length. When he model is close to the maximum power point, it should reduce the step length. The scheme can adapt to the situation of variable wind speed, and the system can react quickly. And the robustness is strong, and the purpose of the experiment is achieved.

\section{References}

[1]. Liu Zhixiong, Wang Yue. Analysis and path selection of China's new energy development [J]. China mining, 2012,06:1-4.

[2]. Abo-Khalil, Ahmed-Lee, Dong-Choon. MPPT control of wind generation systems based on estimated wind speed using SVR[J]. IEEE Transactions on Industrial Electronics. 2008.

[3]. Qi Zhiyuan. Perturbation method and modeling analysis of maximum power control for small wind power generation system and simulation [D]. Inner Mongolia: Inner Mongolia University of Technology, 2005.

[4]. MPPT rapid response control method for wind power generation [J]. Jia Jia Qin, Cao Binggang, Yang Zhongqing. Journal of solar energy. 2004 (02).

[5]. Huang Jincheng, Yang Ping. An optimized maximum power point tracking control algorithm for small wind turbines $[\mathrm{J}]$. motor and control applications. 